\title{
Theory of Elasticity Formulation of the Mindlin Plate Equations
}

\author{
Nwoji, C.U. ${ }^{\# 1}$, Onah H.N. ${ }^{\# 2}$, Mama B.O. ${ }^{\# 3}$, Ike, C.C. $*^{4}$ \\ \#1, \#2, \#3 Dept of Civil Engineering, University of Nigeria, Nsukka, Enugu State, Nigeria. \\ ${ }^{* 4}$ Dept of Civil Engineering \\ Enugu State University of Science \& Technology, Enugu State, Nigeria. \\ 11ifford.nwoji@unn.edu.ng \\ 2hyginus.onah@unn.edu.ng \\ 3benjamin.mama@unn.edu.ng \\ 4ikecc2007@yahoo.com
}

\begin{abstract}
In this work, the mathematical theory of elasticity has been used to formulate and derive from fundamental principles, the first order shear deformation theory originally presented by Mindlin using variational calculus. A relaxation of the Kirchhoff's normality hypothesis was used to account for the effect of the transverse shear strains in the behaviour of the plate. This made the resulting theory appropriate for use for moderately thick plates. A simultaneous use of the strain-displacement relations for small-deformation elasticity, stress-strain laws and the stress differential equations of equilibrium was used to obtain the differential equations of static flexure for Mindlin plates in terms of three unknown generalised displacements. The equations were found to be coupled in the unknown displacements; but reducible to the Kirchhoff plate equations when the removed Kirchhoff normality hypothesis was introduced. This showed the Kirchhoff plate theory to be a specialization of the Mindlin plate theory. The theory of elasticity foundations of the Mindlin and Kirchhoff plate theories are thus highlighted.
\end{abstract}

Keywords: Mindlin plate theory, theory of elasticity, shear deformation.

\section{INTRODUCTION}

\section{BACKGROUND}

Plates are three dimensional structural members bounded by two surfaces: $z= \pm h / 2$, called top and bottom surfaces, and the inplane region on the domain $0 \leq x \leq a, 0 \leq y \leq b$, where the origin is assumed at a corner point ( $x=0, y=0, z=0)$. The distance, $h$, between the top and bottom surfaces, is called the thickness, and this dimension is much smaller than the inplane dimensions $a$, and $b[1,2]$. Generally, plates are categorized into three, namely: thin plates, moderately thick plates and thick plates, depending on the ratio of the thickness to the least inplane dimension $(h / a)$ where $a \leq b[3,4]$. They can also be classified based on the nature of the deformation as small-deformation plates, large deformation plates, or according to their material properties as homogeneous, heterogeneous plates or isotropic, anisotropic plates etc. Plate problems are elasticity problems that are formulated by simultaneous satisfaction of kinematic, material constitutive laws and the differential equations of equilibrium subject to the geometrical and loading conditions [5]. The complete closed form formulation is often difficult, and approximations are commonly employed to reduce the three dimensional elasticity problem to two dimensional approximations.

\section{Plate Theories and Models}

Several theories and models have been formulated to describe plate behaviour. They include Kirchhoff theory, Mindlin [6] theory, Reissner [7] theory, Shimpi [8] refined theory, Reddy [9] theory, Levinson [10] theory, Osadebe [11] plate model, etc. Kirchhoff plate theory, also called the classical small deflection theory of thin plates is the simplest and most commonly used theory of plates. It is a linear infinitesimal theory, suitable for thin isotropic, and orthotropic plates. The equation for static bending of thin istropic plates with variable flexural rigidity is given as:

$$
\nabla^{2}\left(D(x, y) \nabla^{2} w\right)-(1-\mu)\left(\frac{\partial^{2} D}{\partial x^{2}} \frac{\partial^{2} w}{\partial y^{2}}-2 \frac{\partial^{2} D}{\partial x \partial y} \frac{\partial^{2} w}{\partial x \partial y}+\frac{\partial^{2} D}{\partial y^{2}} \frac{\partial^{2} w}{\partial x^{2}}\right)=q_{z}(x, y)
$$

where $D(x, y)$ is the flexural rigidity, $w$ is the transverse deflection, $\nabla^{2}$ is the Laplacian

$$
\nabla^{2}=\frac{\partial^{2}}{\partial x^{2}}+\frac{\partial^{2}}{\partial y^{2}}
$$

$q_{z}(x, y)$ is the distribution of transverse load on the plate, $\mu$ is the Poisson's ratio, and $x$ and $y$ are the inplane Cartesian coordinates. 
The governing equation is a fourth order linear partial differential equation with variable coefficients in $w$, the unknown dependent variable of the equation. For homogeneous plates, the governing equation simplifies to the fourth order equation with constant coefficients given as:

$$
D\left(\frac{\partial^{4} w}{\partial x^{4}}+2 \frac{\partial^{4} w}{\partial x^{2} \partial y^{2}}+\frac{\partial^{4} w}{\partial y^{4}}\right)=D \nabla^{4} w=q_{z}(x, y)
$$

where

$$
\nabla^{4}=\nabla^{2} \nabla^{2}
$$

$\nabla^{4}$ is the biharmonic operator.

Von Karman presented the large deflection thin plate theory as the system of equilibrium and compatibility equations given by:

$$
\begin{aligned}
& \frac{D}{h} \nabla^{4} w(x, y)=L(w, \phi)+\frac{q_{z}}{h} \\
& \frac{1}{E} \nabla^{4} \phi(x, y)=-\frac{1}{2} L(w, w)
\end{aligned}
$$

where

$$
L(w, \phi)=\frac{\partial^{2} w}{\partial x^{2}} \frac{\partial^{2} \phi}{\partial y^{2}}+\frac{\partial^{2} w}{\partial y^{2}} \frac{\partial^{2} \phi}{\partial x^{2}}-2 \frac{\partial^{2} w}{\partial x \partial y} \frac{\partial^{2} \phi}{\partial x \partial y}
$$

$\phi(x, y)$ is the Airy's stress function, $E$ is the Young's modulus of elasticity, $h$ is the plate thickness and

$$
L(w, w)=2 \frac{\partial^{4} w}{\partial x^{2} \partial y^{2}}-2\left(\frac{\partial w}{\partial x \partial y}\right)^{2}
$$

Von Karman's large deflection thin plate theory considers the longitudinal displacement of the middle plane cause by the deflection and the associated stress. The governing equations are coupled, non-linear partial differential equations of fourth order in the dependent variables $w(x, y)$ and $\phi(x, y)$. Kirchhoff classical plate theory, also called the Kirchhoff-Love plate theory was formulated based on the following kinematic assumptions also called the Kirchhoff hypothesis [1-3]

(i) straight lines normal to the middle surface before bending deformation remain straight after bending deformation

(ii) straight lines normal to the mid surface before bending deformation remain normal to the middle surface after bending deformation

(iii) the thickness of the plate does not change during the bending deformation.

The classical Kirchhoff plate theory has been found to give satisfactory results for thin plates, and despite the obvious mathematical simplicity of the governing equations, the theory has the following limitations [12]

(i) the accuracy of the Kirchhoff plate theory decreases with increase in plate thickness, with rapidly increasing or localized forces and in problems of stress concentration around openings in the plate.

(ii) the Kirchhoff plate theory assumes a displacement field for which the strain field is derived. The plate is assumed to be in plane stress state and the stress field derived from the strain field and the stress-strain laws for plane stress conditions. The stress field does not account for the transverse shear stress under the transversely applied load. However, the consideration of equilibrium confirms that the shear stresses in the transverse direction exist.

(iii) since the Kirchhoff plate theory disregards the transverse shear deformation, it results in considerable errors when applied to moderately thick plates for which the transverse shear deformation plays an important role in the behaviour.

(iv) the Kirchhoff plate theory uses an incomplete set of boundary conditions resulting in errors especially around the plate boundaries, as well as in support reactions.

These limitations of the Kirchhoff plate theory have led to the development of other plate theories in order to address some of the lapses of the Kirchhoff plate theory. Some of these plate theories developed in answer to the limitations of the Kirchhoff plate theory are Reissner plate theory, Mindlin plate theory, Reddy plate theory, Levinson's plate theory, Shimpi's refined plate theory, etc. In this paper, a systematic formulation of the Mindlin plate theory is done using the methods of the theory of elasticity. The formulation of the Mindlin plate equations uses a displacement based approach. 


\section{RESEARCH Aim AND OBJECTIVES}

The research aim is to present a theory of elasticity formulation of the Mindlin plate equations for static flexure using the equilibrium approach. The objectives are:

(i) to obtain the displacement field, the strain field, and the stress fields over the plate due to transverse load;

(ii) to obtain the internal force resultants;

(iii) to use the differential equations of equilibrium and obtain the internal force resultants in terms of the generalized displacements;

(iv) to obtain the governing partial differential equations from a consideration of the differential equations of equilibrium.

\section{THEORETICAL Framework}

Plate problems of static flexure and dynamic flexure are elasticity problems, and are formulated by simultaneously considering the strain-displacement (kinematic) relations, the material constitutive (stress-strain) laws, and the differential equations of equilibrium.

\section{KinEMATIC RELATIONS}

The kinematic relations for small-displacement assumptions are:

$$
\begin{aligned}
& \varepsilon_{x x}=\frac{\partial u}{\partial x} \\
& \varepsilon_{y y}=\frac{\partial v}{\partial y} \\
& \varepsilon_{z z}=\frac{\partial w}{\partial z} \\
& \gamma_{x y}=\frac{\partial u}{\partial y}+\frac{\partial v}{\partial x} \\
& \gamma_{y z}=\frac{\partial v}{\partial z}+\frac{\partial w}{\partial y} \\
& \gamma_{x z}=\frac{\partial u}{\partial z}+\frac{\partial w}{\partial x}
\end{aligned}
$$

where $\varepsilon_{x x}, \varepsilon_{y y}, \varepsilon_{z z}$ are normal strains, $\gamma_{x y}, \gamma_{x z}$, and $\gamma_{y z}$ are shear strains, $u(x, y, z), v(x, y, z)$ and $w(x, y, z)$ are components of the displacement field in the $x, y$, and $z$ Cartesian coordinate directions.

\section{STRESS-STRAIN LAWS}

The stress-strain laws for homogeneous isotropic materials are:

$$
\begin{aligned}
& \varepsilon_{x x}=\frac{1}{E}\left(\sigma_{x x}-\mu \sigma_{y y}-\mu \sigma_{z z}\right) \\
& \varepsilon_{y y}=\frac{1}{E}\left(\sigma_{y y}-\mu \sigma_{x x}-\mu \sigma_{z z}\right) \\
& \varepsilon_{z z}=\frac{1}{E}\left(\sigma_{z z}-\mu \sigma_{x x}-\mu \sigma_{y y}\right) \\
& \gamma_{x y}=\frac{\tau_{x y}}{G}=\frac{2(1+\mu)}{E} \tau_{x y} \\
& \gamma_{y z}=\frac{\tau_{y z}}{G}=\frac{2(1+\mu)}{E} \tau_{y z} \\
& \gamma_{z x}=\frac{\tau_{z x}}{G}=\frac{2(1+\mu)}{E} \tau_{z x}
\end{aligned}
$$

where $\sigma_{x x}, \sigma_{y y}, \sigma_{z z}$ are normal stresses, $\tau_{x y}, \tau_{y z}, \tau_{z x}$ are shear stresses, $E$ is the Young's modulus of elasticity, $\mu$ is the Poisson's ratio and $G$ is the shear modulus. 


\section{DIFFERENTIAL EQUATIONS OF STATIC EQUILIBRIUM}

The differential equations of static equilibrium of an infinitesimal plate element; in the absence of body forces are given in terms of stresses as:

$$
\begin{aligned}
& \frac{\partial \sigma_{x x}}{\partial x}+\frac{\partial \sigma_{y x}}{\partial y}+\frac{\partial \sigma_{z x}}{\partial z}=0 \\
& \frac{\partial \sigma_{y x}}{\partial x}+\frac{\partial \sigma_{y y}}{\partial y}+\frac{\partial \sigma_{y z}}{\partial z}=0 \\
& \frac{\partial \sigma_{z x}}{\partial x}+\frac{\partial \sigma_{z y}}{\partial y}+\frac{\partial \sigma_{z z}}{\partial z}=0
\end{aligned}
$$

In terms of internal force resultants, the equations are:

$$
\begin{aligned}
& \frac{\partial Q_{x}}{\partial x}+\frac{\partial Q_{y}}{\partial y}+q_{z}(x, y)=0 \\
& Q_{x}=\frac{\partial M_{x x}}{\partial x}+\frac{\partial M_{x y}}{\partial y} \\
& Q_{y}=\frac{\partial M_{y y}}{\partial y}+\frac{\partial M_{x y}}{\partial x}
\end{aligned}
$$

where $Q_{x}$, and $Q_{y}$ are shear force resultants $M_{x x}, M_{y y}$, are the bending moments and $M_{x y}$ is the twisting moment.

\section{Theory of Elasticity Formulation of Mindlin Plate Equations}

\section{FUNDAMENTAL ASSUMPTIONS}

The following assumptions are made:

(i) The plate deflection under transverse loads are small and finite, hence small displacement relations of elasticity theory are valid.

(ii) Normals to the plate undeformed middle surface before deformation remain straight and unstretched in length but they are not necessarily normal to the plate middle surface after deformation. The implication of the relaxation of the normality rule is that the shear strains $\varepsilon_{x z}$ and $\varepsilon_{y z}$ are not zero, and the Mindlin theory of plates considers the effect of shear deformation.

(iii) Stresses normal to the plate middle surface are negligible, thus

$$
\sigma_{z z}=0
$$

(iv) There is no deformation in the middle surface of the plate, which remains a neutral surface.

(v) The state of deformation is described completely by the transverse displacement in the $z$ direction of the middle surface $w(x, y, z=0)$, and $\theta_{x}(x, y), \theta_{y}(x, y)$ where $\theta_{x}, \theta_{y}$ are the rotations about the $x$ and $y$ axes of lines normal to the middle surface before deformation.

\section{THEORY OF ELASTICITY FORMULATION OF THE MINDLIN PLATE EQUATIONS}

The theory of elasticity formulation of Mindlin plate equations for static flexure is based on the kinematic relations, the material constitutive relations, and the differential equations of static equilibrium.

\section{DISPLACEMENT FIELD}

The displacement field at a generic point $(x, y, z)$ of the plate is given by the deflections of the plate middle surface as follows:

$$
\begin{aligned}
& w(x, y, z)=w(x, y, z=0)=w(x, y) \\
& u(x, y, z)=u(x, y, z=0)=z \theta_{x}(x, y) \\
& v(x, y, z)=v(x, y, z=0)=z \theta_{y}(x, y)
\end{aligned}
$$

where $u, v$, and $w$ are the components of the displacement field in the $x, y$, and $z$ coordinate directions, respectively. 


\section{STRAIN FIELDS}

The strain fields are obtained using the kinematic relations as follows:

$$
\begin{aligned}
& \varepsilon_{x x}=z \frac{\partial \theta_{x}}{\partial x} \\
& \varepsilon_{y y}=z \frac{\partial \theta_{y}}{\partial y} \\
& \varepsilon_{z z}=0 \\
& \gamma_{x y}=z\left(\frac{\partial \theta_{x}}{\partial y}+\frac{\partial \theta_{y}}{\partial x}\right) \\
& \gamma_{x z}=\theta_{x}+\frac{\partial w}{\partial x} \\
& \gamma_{y z}=\theta_{y}+\frac{\partial w}{\partial y}
\end{aligned}
$$

\section{STRESS-STRAIN LAWS}

The stress-strain laws for the case when $\sigma_{z z}=0$ are; for isotropic, homogeneous materials,

$$
\begin{aligned}
& \sigma_{x x}=\frac{E}{1-\mu^{2}}\left(\varepsilon_{x x}+\mu \varepsilon_{y y}\right) \\
& \sigma_{y y}=\frac{E}{1-\mu^{2}}\left(\varepsilon_{y y}+\mu \varepsilon_{x x}\right) \\
& \tau_{x y}=G \gamma_{x y}=\frac{E}{2(1+\mu)} \gamma_{x y} \\
& \tau_{x z}=G \gamma_{x z}=\frac{E}{2(1+\mu)} \gamma_{x z} \\
& \tau_{y z}=G \gamma_{y z}=\frac{E}{2(1+\mu)} \gamma_{y z}
\end{aligned}
$$

\section{STRESS FIELDS (OR STRESS DISPLACEMENT EQUATIONS)}

The strain fields are substituted into the stress-strain laws to obtain the stress fields as follows:

$$
\begin{aligned}
\sigma_{x x} & =\frac{E z}{1-\mu^{2}}\left(\frac{\partial \theta_{x}}{\partial x}+\mu \frac{\partial \theta_{y}}{\partial y}\right) \\
\sigma_{y y} & =\frac{E z}{1-\mu^{2}}\left(\frac{\partial \theta_{y}}{\partial y}+\mu \frac{\partial \theta_{x}}{\partial x}\right) \\
\tau_{x y} & =\frac{E z}{2(1+\mu)}\left(\frac{\partial \theta_{x}}{\partial y}+\frac{\partial \theta_{y}}{\partial x}\right) \\
\tau_{x z} & =G\left(\frac{\partial w}{\partial x}+\theta_{x}\right) \\
\tau_{y z} & =G\left(\frac{\partial w}{\partial y}+\theta_{y}\right)
\end{aligned}
$$

\section{INTERNAL FORCE RESULTANTS} thickness:

The internal force resultants $M_{x x}, M_{y y}, M_{x y}, Q_{x}$ and $Q_{y}$ are obtained as the following integrals over the plate

$$
M_{x x}=\int_{-h / 2}^{h / 2} \sigma_{x x} z d z
$$




$$
\begin{aligned}
& M_{y y}=\int_{-h / 2}^{h / 2} \sigma_{y y} z d z \\
& M_{x y}=\int_{-h / 2}^{h / 2} \tau_{x y} z d z \\
& Q_{x}=\int_{-h / 2}^{h / 2} \tau_{x z} d z \\
& Q_{y}=\int_{-h / 2}^{h / 2} \tau_{y z} d z
\end{aligned}
$$

where $M_{x x}, M_{y y}$ are the bending moments, $M_{x y}$ is the twisting moment, $Q_{x}$ and $Q_{y}$ are shear forces.

By substitution of the strain fields, and integration of the resulting expressions with respect to the $z$ coordinate, we obtain the internal force resultants as follows:

$$
\begin{aligned}
& M_{x x}=\int_{-h / 2}^{h / 2} \frac{E z^{2}}{1-\mu^{2}}\left(\frac{\partial \theta_{x}}{\partial x}+\mu \frac{\partial \theta_{y}}{\partial y}\right) d z \\
& M_{x x}=D\left(\frac{\partial \theta_{x}}{\partial x}+\mu \frac{\partial \theta_{y}}{\partial y}\right) \\
& M_{y y}=\int_{-h / 2}^{h / 2} \frac{E z^{2}}{1-\mu^{2}}\left(\frac{\partial \theta_{y}}{\partial y}+\mu \frac{\partial \theta_{x}}{\partial x}\right) d z \\
& M_{y y}=D\left(\frac{\partial \theta_{y}}{\partial y}+\mu \frac{\partial \theta_{x}}{\partial x}\right) \\
& M_{x y}=\int_{-h / 2}^{h / 2} \frac{E z^{2}}{2(1+\mu)}\left(\frac{\partial \theta_{x}}{\partial y}+\frac{\partial \theta_{y}}{\partial x}\right) d z \\
& M_{x y}=\frac{D(1-\mu)}{2}\left(\frac{\partial \theta_{x}}{\partial y}+\mu \frac{\partial \theta_{y}}{\partial x}\right)
\end{aligned}
$$

Shear force correction coefficients $k$ are introduced into the definition for the shear force expressions in order to consider the parabolic distribution of shear stresses in the $z$ direction. Applying the shear correction coefficient (factor),

$$
\begin{aligned}
& Q_{x}=k \int_{-h / 2}^{h / 2} \tau_{x z} d z=k \int_{-h / 2}^{h / 2} G\left(\theta_{x}+\frac{\partial w}{\partial x}\right) d z \\
& Q_{x}=k G h\left(\theta_{x}+\frac{\partial w}{\partial x}\right)=D_{Q}\left(\theta_{x}+\frac{\partial w}{\partial x}\right) \\
& Q_{y}=k \int_{-h / 2}^{h / 2} \tau_{y z} d z=k \int_{-h / 2}^{h / 2} G\left(\theta_{y}+\frac{\partial w}{\partial y}\right) d z \\
& Q_{y}=k G h\left(\theta_{y}+\frac{\partial w}{\partial y}\right)=D_{Q}\left(\theta_{y}+\frac{\partial w}{\partial y}\right)
\end{aligned}
$$

\section{DIFFERENTIAL EQUATIONS OF STATIC EQUILIBRIUM}

The differential equations of static equilibrium for a differential element of the plate when body forces are absent are Equations (21) - (23). In terms of internal force resultants, the differential equations of equilibrium are Equations (24) - (26). The three differential equations of equilibrium can be expressed as a single equation as follows:

$$
\frac{\partial^{2} M_{x x}}{\partial x^{2}}+2 \frac{\partial^{2} M_{x y}}{\partial x \partial y}+\frac{\partial^{2} M_{y y}}{\partial y^{2}}+q_{z}(x, y)=0
$$

By substitution of expressions obtained for $M_{x x}, M_{x y}$, and $M_{y y}$, the differential equation of equilibrium become: 


$$
D\left(\frac{\partial^{3} \theta_{x}}{\partial x^{3}}+\mu \frac{\partial^{3} \theta_{y}}{\partial x^{2} \partial y}\right)+D\left(\frac{\partial^{3} \theta_{y}}{\partial y^{3}}+\mu \frac{\partial^{3} \theta_{x}}{\partial x \partial y^{2}}\right)+\frac{2 D(1-\mu)}{2}\left(\frac{\partial^{3} \theta_{x}}{\partial x \partial y^{2}}+\frac{\partial^{3} \theta_{y}}{\partial x^{2} \partial y}\right)+q_{z}(x, y)=0
$$

Simplifying,

$$
D \nabla^{2}\left(\frac{\partial \theta_{x}}{\partial x}+\frac{\partial \theta_{y}}{\partial y}\right)=-q_{z}(x, y)
$$

Also, from Equation (24),

$$
k G h \frac{\partial}{\partial x}\left(\theta_{x}+\frac{\partial w}{\partial x}\right)+k G h \frac{\partial}{\partial y}\left(\theta_{y}+\frac{\partial w}{\partial y}\right)=-q
$$

Simplifying,

$$
\nabla^{2} w+\frac{\partial \theta_{x}}{\partial x}+\frac{\partial \theta_{y}}{\partial y}=-\frac{q}{k G h}=-\frac{q}{D_{Q}}
$$

From Equation (25), we obtain upon substitution,

$$
D\left(\frac{\partial^{2} \theta_{x}}{\partial x^{2}}+\mu \frac{\partial^{2} \theta_{y}}{\partial x \partial y}\right)+\frac{1-\mu}{2}\left(\frac{\partial^{2} \theta_{x}}{\partial y^{2}}+\frac{\partial^{2} \theta_{y}}{\partial y \partial x}\right)=k G h\left(\theta_{x}+\frac{\partial w}{\partial x}\right)
$$

After simplification,

$$
\frac{D}{2}\left((1-\mu) \nabla^{2} \theta_{x}+(1+\mu) \frac{\partial}{\partial x}\left(\frac{\partial \theta_{x}}{\partial x}+\frac{\partial \theta_{y}}{\partial y}\right)\right)=Q_{x}=k G h\left(\theta_{x}+\frac{\partial w}{\partial x}\right)
$$

Similarly, from Equation (26), we obtain, upon substitution,

$$
\frac{D(1-\mu)}{2}\left(\frac{\partial^{2} \theta_{x}}{\partial x \partial y}+\frac{\partial^{2} \theta_{y}}{\partial x^{2}}\right)+D\left(\frac{\partial^{2} \theta_{y}}{\partial y^{2}}+\mu \frac{\partial^{2} \theta_{x}}{\partial x \partial y}\right)=Q_{y}=k G h\left(\theta_{y}+\frac{\partial w}{\partial y}\right)
$$

After simplification,

$$
\begin{aligned}
& \frac{D}{2}\left((1-\mu) \nabla^{2} \theta_{y}+(1+\mu) \frac{\partial}{\partial y}\left(\frac{\partial \theta_{x}}{\partial x}+\frac{\partial \theta_{y}}{\partial y}\right)\right)=Q_{y}=k G h\left(\theta_{y}+\frac{\partial w}{\partial y}\right) \\
& \text { Let } \quad \frac{\partial \theta_{x}}{\partial x}+\frac{\partial \theta_{y}}{\partial y}=M=\frac{M_{s}}{D}
\end{aligned}
$$

where $M_{s}$, the Marcus moment, is defined as:

$$
M_{s}=\frac{M_{x x}+M_{y y}}{1+\mu}
$$

Then, the governing partial differential equations of Mindlin plates are:

$$
\begin{aligned}
& \frac{D}{2}\left((1-\mu) \nabla^{2} \theta_{x}+(1+\mu) \frac{\partial M}{\partial x}-D_{Q}\left(\theta_{x}+\frac{\partial w}{\partial x}\right)\right)=0 \\
& \frac{D}{2}\left((1-\mu) \nabla^{2} \theta_{y}+(1+\mu) \frac{\partial M}{\partial y}-D_{Q}\left(\theta_{y}+\frac{\partial w}{\partial y}\right)\right)=0 \\
& D_{Q}\left(\nabla^{2} w+M\right)+q_{z}(x, y)=0
\end{aligned}
$$

When

$$
\gamma_{x z}=0
$$

i.e. $\quad \theta_{x}=-\frac{\partial w}{\partial x}$

and

$$
\begin{aligned}
& \gamma_{y z}=0 \\
& \theta_{y}=-\frac{\partial w}{\partial y}
\end{aligned}
$$


The Mindlin plate equations become:

$$
\begin{aligned}
& M_{x x}=-D\left(\frac{\partial^{2} w}{\partial x^{2}}+\mu \frac{\partial^{2} w}{\partial y^{2}}\right) \\
& M_{y y}=-D\left(\frac{\partial^{2} w}{\partial y^{2}}+\mu \frac{\partial^{2} w}{\partial x^{2}}\right) \\
& M_{x y}=-D(1-\mu)\left(\frac{\partial^{2} w}{\partial x \partial y}\right) \\
& \nabla^{2} \nabla^{2} w=\nabla^{4} w=\frac{q_{z}}{D} \\
& Q_{x}=0 \\
& Q_{y}=0
\end{aligned}
$$

\section{Discussion}

This study has successfully presented the first principles formulation and derivation of the Mindlin first order shear deformation plate theory using the methods of the theory of elasticity. The kinematics assumptions of the classical Kirchhoff plate theory was extended by removing the normality restrictions, thereby including the transverse shear strains. The transverse shear strains were found from Equations $(45,46)$ to be constant through the plate thickness, whereas the stress-equilibrium equations predict a quadratic (parabolic) variation of the transverse shear stresses over the plate thickness. In order to make the shear forces $\left(Q_{x}, Q_{y}\right)$ computed in the present study to be equal to those obtained using the parabolic variation of transverse shear stresses obtained from the stress equilibrium equations, shear correction factors were introduced in the definition of the internal shear stress resultants (Equation (58) and (60)).

In the derivation of the stress-strain equations of the plate, plane stress state assumption is made, similar to the classical Kirchhoff zeroth order shear deformation plate theory. The plane stress form of the stress-strain law Equations (37-41) is used. In both the classical Kirchhoff zeroth order shear deformation plate theory and the Mindlin first order shear deformation plate theory, the assumption of inextensibility of the middle plane yielding $\varepsilon_{z z}=0$, and/or straightness of transverse normals can be removed. Such extensions of the assumptions of the present work can yield second order and higher order shear deformation models of plates under static, and/or dynamic flexure. When the transverse shear stresses are made to be zero, i.e. $\theta_{x}=-\partial w / \partial x$ and $\theta_{y}=-\partial w / \partial y$, the equations of the Mindlin plate theory reduce to the equations of the classical Kirchhoff zeroth order shear deformation plate theory. Bending moment resultants were obtained from a simultaneous consideration of kinematics and stress-strain laws as Equations (53) and (55). The twisting moment resultant was similarly obtained as Equation (57). The shear force resultants were found, similarly as Equations (59) and (61). The system of differential equations of static equilibrium expressed in terms of stress resultants were then used, together with the internal force resultants obtained from the simultaneous consideration of the kinematic assumptions, and the stress-strain laws to obtain the governing differential equations of equilibrium of Mindlin plates as Equations (64), (68) and (70) or alternatively as Equations (73) - (75). The equations are a system of three coupled partial differential equations in the three unknown variables $w(x, y), \theta_{x}(x, y)$ and $\theta_{y}(x, y)$.

Despite the many obvious merits of the Mindlin plate theory, it suffers the following limitations:

(i) the theory yields a constant shear strain distribution over the plate thickness, in violation of the known parabolic distribution of shear strain obtained in a rigorous application of theory of elasticity. The introduction of the shear stress correction factor merely ensures that the resultant shear stress agrees with the predictions of the theory of elasticity, but the distributions of the shear stress over the plate thickness are in violation of the theory of elasticity.

(ii) the shear free boundary condition on the plate surface is violated

(iii) the theory is two-dimensional and cannot correctly predict practical three dimensional plates.

\section{Conclusions}

The conclusions of the study are as follows:

(i) the governing partial differential equations of equilibrium of Mindlin plates have been successfully derived using a displacement approach in the theory of elasticity. 
(ii) the governing equations of Mindlin plates, were derived from a simultaneous consideration of the kinematic equations, stress-strain laws and differential equations of equilibrium and thus simultaneously satisfy the kinematic, stress-strain laws, as well as the equilibrium equations of elasticity theory.

(iii) the governing equations are a system of three coupled partial differential equations in terms of three unknown generalized displacements, and can be solved mathematically.

(iv) the Mindlin plate equations simplify to the classical Kirchhoff zeroth order shear deformation plate equations when the normality conditions are strictly imposed in the kinematic equations; making the Kirchhoff plate theory a special case of the Mindlin plate theory.

(v) the Kirchhoff plate theory is easily derivable from the Mindlin plate theory when $\theta_{x}=-\partial w / \partial x$, $\theta_{y}=-\partial w / \partial y$.

\section{REFERENCES}

[1] J.N. Reddy “Theory and Analysis of Elastic Plates,” Taylor and Francis, London, 1999.

[2] K. Chandrashekhara “Theory of Plates,” Universities Press (India) Limited Hyderabad, 2011.

[3] R. Szilard "Theories and Applications of Plate Analysis: Classical, Numerical and Engineering Methods," John Wiley and Sons Inc, New Jersey, 2004.

[4] Ashwin Balasubramanian "Plate Analysis with Different Geometries and Arbitrary Boundary Conditions,” MSc Thesis, Mechanical Engineering, Faculty of Graduate School, the University of Texas at Arlington, December 2011.

[5] V.K. Sebastian “An Elastic Solution for Simply Supported Rectangular Plates," Nigerian Journal of Technology (NIJOTECH) Vol.7 No1, pp 11-16, September, 1983

[6] R.D. Mindlin "Influence of Rotary Inertia on Flexural Motion of Isotropic, Elastic Plates” Journal of Applied Mechanics Vol. 18 No 1, pp. $31-38,1945$.

[7] E. Reissner "The effect of shear deformation on the bending of elastic plates," Journal of Applied Mechanics, Vol 12 pp A69, 1945.

[8] R.P. Shimpi “Refined Plate Theory and its Variants,” AIAA Journal, Vol 40, pp. 137-146. 2002.

[9] J.N. Reddy "A refined non-linear theory of plates with transverse shear deformation," International Journal of Solids and Structures, Vol. 20 pp. 881-896, 1984.

[10] M. Levinson "An accurate simple theory of statics and dynamics of elastic plates," Mechanics Research Communications, Vol.7, pp. 343-350, 1980.

[11] N.N. Osadebe "Differential equations of small deflection analysis of thin elastic plates possessing extensible middle surface," University of Nigeria Virtual Library, 1997.

[12] C.C. Ike "Equilibrium Approach in the Derivation of Differential Equations for Homogeneous Isotropic Mindlin Plates," Nigerian Journal of Technology (NIJOTECH) Vol 36, No.2, pp 346-350, April 2017.

\section{Author Profile}

Engr Dr. Clifford U. Nwoji obtained his civil engineering education from University of Nigeria Nsukka. He is a member of Nigerian Society of Engineers and also a registered engineer with Council for Regulation of Engineering in Nigeria. He has substantial industrial experience. Since obtaining his higher degrees in engineering he has been teaching structural engineering courses at both undergraduate and postgraduate levels. He has successfully supervised higher degree theses. He has to his credit a good number of published research papers in reputable international journals.

Hyginus Nwankwo Onah (B.Eng, M.Eng, PhD) Born in Nigeria on May $4^{\text {th }} 1959$, I obtained my B.Eng. (Civil Engineering) in 1983. After brief work in Lagos Nigeria, I travelled to France in 1987 where I obtained M.Eng (Civil Engineering) at INSA de Lyon and Ph.D (Structural Mechanics) at CNAM Paris in 1991. Then I worked as a researcher at Research and Development (R\&D) unit of RENAULT Motors Reuil Malmaison France, Department of Engineering University of Manchester England and Becchis Osiride of FIAT Motors Torino Italy for one year each. From January 1996 to date as a lecturer in Civil Engineering Department of University of Nigeria, Nsukka, I teach and supervise both undergraduate and Postgraduate students and carry out research activities in mechanics and behavior of structures

Engr Dr Benjamin Okwudili Mama I got my PhD in the year 2009 in the University of Nigeria, Nsukka. My master's degree in civil Structural Engineering in 1992 in the same School. My first degree in Civil Engineering in then Anambra State University of Technology, Enugu. I have been lecturing in the Department of Civil Engineering, University of Nigeria, Nsukka from 1994 till date.

Engr Dr. Charles C. Ike is a lecturer at the Enugu State University of Science and Technology, Enugu, Nigeria. He holds a PhD in structural engineering, and is a member of the Nigerian Society of Engineers. He is registered with the Council for the Regulation of Engineering in Nigeria (COREN). He has numerous publications in reputable international journals. 